\title{
Modern trends in Green's functions: theory and applications
}

The purpose of this issue is to highlight the achievements in the development of Green's functions (GF) method and its applications in recent years. Despite a rather long history of the method, it remains a powerful one and is successfully used in considering the many-particle interactions in problems of statistical physics. A new impulse to its development in the theory of equilibrium systems has been given in the last years by the intense outburst of investigations in the field of strongly correlated electron systems. One can mention, in particular, an elaboration of the dynamical mean field theory, the renascence of Kadanoff and Baym ideas and approach. Recent developments of the GF method are connected with consideretaion of systems where the simple diagram technique for the Matsubara GF of the Fermi- Boze-operators cannot be applyed. This results in developing of some kind of self-consistent calculations, as the equation of motion method, the generating functional approach, etc. The objects of investigations are high- $T_{\mathrm{c}}$ superconductors, valence change compounds, Mott-Hubbard dielectrics etc.

Our task is also to elucidate a modern state of the method of non-equilibrium GF and its development in application to the kinetic theory, thermo-field dynamics, application of GF to non-equilibrium phenomena in condensed matter and description of many-particle correlations in relaxation processes.

The papers in this collection are grouped according to their main focus. The first part is composed of papers devoted to the equilibrium GF. Review of the renormalization methods in the many-body perturbation theory is given by V. Janiš. The way of constructing the LattingerWard functional is developed by M. Potthoff. The ergodicity issue in strongly correlated systems is considered by F. Mancini and A. Avella using Green's function formalism; the question of nonergodicity in spectral relations for multi-time correlation functions is discussed by A.M. Shvaika. The analysis of the spectrum of the s-d-exchange model within the framework of the generating functional approach is given by Yu.A. Izyumov, N.I. Chaschin and D.S. Alexeev. Such an approach is also extended to the dynamical mean field theory and applied to the asymmetric Hubbard model by I.V. Stasyuk and O.B. Hera. F. Mancini and A. Avella give a brief review of the composite operator method in the theory of strongly correlated electron systems. A formulation of Green's function method in the theory of superconductivity for various types of pairing is considered by N.M. Plakida. An application of the method to the more elaborate study of the Hubbard model spectrum is made by A. Sherman.

In the second part of the issue the papers are collected, which are connected with the method of non-equilibrium Green's functions. An extended quasiparticle approximation for relativistic electrons in plasma within the path-ordered Green's function formalism is developed by V.G. Morozov and G. Röpke. Short-time kinetics and initial correlations in quantum systems are considered by D. Kremp, D. Semkat and Th. Bornath. The theory of nonlinear Peltier effect in relation to the nonequilibrium Jonson-Mahan theorem is given by Y.K. Freericks and V. Zlatić. The Green's functions of systems with bound states and the response to the external electromagnetic field are analyzed by Yu.V. Slyusarenko and A.G. Sotnikov. A reduced description of the nonequilibrium processes and time correlation functions is proposed by A.I. Sokolovsky. Nonequilibrium properties of inhomogeneous electron gas are studied by P. Kostrobii, B. Markovych, A. Vasylenko, M. Tokarchuk and Yu. Rudavskii using the Zubarev method of nonequilibrium statistical operator and the Green's functions approach. 
We express our gratitude to all the authors for their readiness to participate in the issue and for their cooperation. We also thank Vasyl Ignatyuk, Andrij Shvaika and Taras Mysakovych for their help and technical assistance.

V. Morozov, N. Plakida, I. Stasyuk 